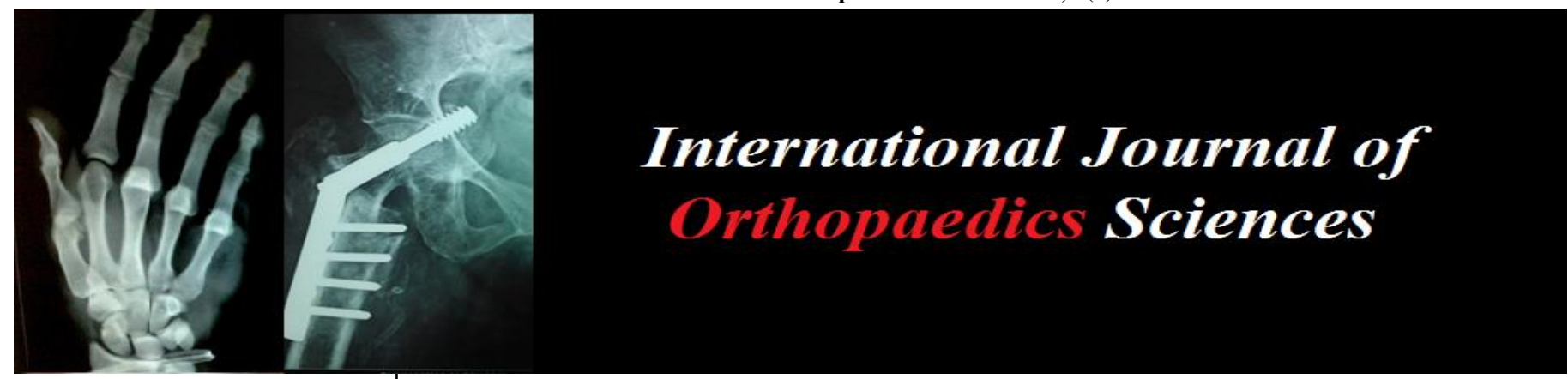

E-ISSN: 2395-1958

P-ISSN: 2706-6630

IJOS 2020; 6(2): 510-512

(C) 2020 IJOS

www.orthopaper.com

Received: 26-02-2020

Accepted: 28-03-2020

Dr. Waghmare Gaurav Seth GS Medical College and

KEM Hospital, Acharya Donde

Marg, Parel, Mumbai,

Maharashtra, India

Dr. Garg Bipul Kumar

Seth GS Medical College and

KEM Hospital, Acharya Donde

Marg, Parel, Mumbai,

Maharashtra, India

Dr. Banerjee Subhashis

Seth GS Medical College and

KEM Hospital, Acharya Donde

Marg, Parel, Mumbai,

Maharashtra, India

Dr. Gautham Suresh

Seth GS Medical College and

KEM Hospital, Acharya Donde

Marg, Parel, Mumbai,

Maharashtra, India
Corresponding Author: Dr. Waghmare Gaurav Seth GS Medical College and KEM Hospital, Acharya Donde Marg, Parel, Mumbai,

Maharashtra, India

\section{Plantar fasciitis: Evidence-based review of current treatment modalities}

\author{
Dr. Waghmare Gaurav, Dr. Garg Bipul Kumar, Dr. Banerjee Subhashis \\ and Dr. Gautham Suresh
}

DOI: https://doi.org/10.22271/ortho.2020.v6.i2h.2092

\section{Abstract}

Various evidence based treatment options are available for the treatment of plantar fasciitis. This article is a review of recent literature comparing various treatment modalities used for the treatment of plantar fasciitis. This will assist health care providers to make a sound diagnosis and treatment of heel pain.

Keywords: Various evidence evidence-based current treatment modalities

\section{Introduction}

The plantar fascia is a thick fibrous aponeurosis with its origin at the calcaneus and insertion on the tendons of forefoot and proximal phalanges. It supports the foot and acts as a shock absorber for pressure placed on the foot ${ }^{[1,2]}$.

A series of repetitive micro-trauma to the plantar fascia causes inflammation and eventual degradation thus causing plantar fasciitis ${ }^{[1]}$.

Plantar fasciitis affects both sedentary and athletic populations ${ }^{[3]}$.

The mechanism by which the body heals a chronic degeneration is a mystery which makes the treatment very challenging.

\section{Discussion}

The term plantar fasciitis is most recently been replaced by the term plantar fasciosis since inflammation is not the major cause of the pain ${ }^{[4]}$.

Symptoms include severe pain in the morning or after prolonged rest and improves on movement. Local examination findings are tenderness along the medial part of the heel, discomfort with passive dorsiflexion of first toe and sometimes a tightness of the Achilles tendon or the gastrocnemius muscle ${ }^{[5]}$. Risk factors for plantar fasciitis include excessive foot pronation or flat feet (pes planus), high arches (pes cavus), tight Achilles tendon or gastrocnemius muscle (equinus), tight intrinsic foot muscles, limb length discrepancy, obesity, running, prolonged standing or walking, poor-fitting shoes, and improper gait ${ }^{[4]}$.

\section{Diagnosis}

Plantar fasciitis is most often a straight forward clinical diagnosis and imaging can only confirm or rule out other causes of heel pain. Ultrasound is another relatively inexpensive diagnostic tool that can rule out certain causes of heel pain (such as plantar fibromatosis, foreign body, and plantar xanthomas) and can aid in diagnosis by establishing plantar fascial thickness and the presence of fascial tears ${ }^{[6]}$. Various studies have shown that in patients with symptomatic plantar fasciitis, the plantar fascia thickness tends to be greater than $4 \mathrm{~mm}$ on diagnostic ultrasound ${ }^{[7]}$.

\section{Treatment}

The pain is self-limiting and resolves within a year but the constant hinderance to activities of daily living prompts the patients to seek treatment before the pain resolves. Conservative treatment is effective in $70 \%$ to $80 \%$ of patients ${ }^{[1]}$ however a combination of therapies is usually used ${ }^{[4]}$. 
Plantar fasciitis is initially treated with a combination of rest, ice fomentation, NSAIDs [4] and sometimes with acetaminophen or corticosteroids, however these often provide only temporary relief ${ }^{[4]}$.

\section{Stretching}

Plantar fasciitis as many believe may be caused by tight gastrocnemius and intrinsic foot muscles ${ }^{[8]}$.

A physical therapy stretching programme is a low cost and effective method comprising of plantar fascia and heel cord stretching excercises which can even be done in an at-home basis.

\section{Orthotics}

Orthotics with heel cups and arch supports are believed to treat the underlying biomechanics of plantar fasciitis such as flat feet, high arches and thereby reducing the peak plantar pressure ${ }^{[2]}$. Night splints worn while patients are sleeping prevents the foot from resting in a plantar-flexed position, increases calf and plantar muscle flexibility and reduces tension on the plantar fascia.

However night splints maybe cumbersome and limit patient adherence ${ }^{[9]}$. In one study the combined use of orthotics and night splints yielded better results in treating heel pain, compared with orthotics alone ${ }^{[2]}$.

\section{Invasive treatments}

Corticosteroid injections are one of the most popular invasive treatments for plantar fasciitis. Other types of injections include platelet-rich plasma and autologous blood. Although invasive, injections have few complications, most commonly seen are post injection pain and (rarely) fat pad atrophy, infection, nerve injury, and plantar fascia rupture ${ }^{[10]}$. A study compared corticosteroid injections with placebo and determined that corticosteroid injections provided short-term relief lasting no more than 1 month ${ }^{[10]}$. Corticosteroid injections can be costly and increase the risk of plantar fascia rupture. For these reasons, they mainly are used as short-term treatment, though some providers consider them first-line ${ }^{[10]}$. A study comparing corticosteroid injections with platelet-rich plasma (PRP) injections and found no significant differences in outcomes ${ }^{[11]}$.

Autologous blood injections are a newer treatment and have not yet been studied thoroughly. One of the few studies revealed that $85 \%$ of patients had improvement and $68 \%$ remained pain-free at 12-month follow-up ${ }^{[12]}$.

\section{Other treatment options}

Extracoporeal shock wave therapy (ESWT) produces high energy sound waves that promote healing and growth factor incorporation in the degenerated fascia. In a recent study of ESWT, patients were given between four and eleven sessions of therapy, with an average of 2,000 pulses per session. The results were significant reduction of pain at 1 month, 3 months, and 1 year, with a pain intensity rating that continued to decrease as time went on. Treatment success rate was $98 \%$ at 1 year (measured as at least a $60 \%$ reduction in pain) with an $8 \%$ rate of symptom recurrence ${ }^{[6]}$.

Local radiation for the treatment of plantar fasciitis decreases the inflammatory mediators and pain. In a study comparing corticosteroid injections with radiation therapy, patients received radiation 3 days per week for 2 weeks with followup at 3 and 6 months. At both follow-ups, patients who received radiation therapy had significantly lower pain scores than those who received corticosteroid injections. Radiation is considered as a treatment option if other treatments fail ${ }^{[13]}$. Surgical treatment is the last option which includes partial or complete plantar fascia release and gastrocnemius release The results after surgery are highly variable ${ }^{[1]}$.

Complications like infection, nerve injury and fascia rupture are not uncommon. Recovery can take as long as 3 months and still may not have complete resolution of pain. Monteagudo and colleagues found that $60 \%$ of patients who had partial plantar fasciotomy had symptoms improvement, compared with $95 \%$ of those who had complete gastrocnemius release. Additionally, the patients with a proximal release had an average recovery of 10 weeks, compared with 3 weeks for patients with gastrocnemius resection ${ }^{[14]}$.

Surgery may be the last option used for the treatment of unresponsive plantar fasciitis and should be discussed in detail with the patient regarding the expected outcomes and possible complications.

\section{Conclusion}

Plantar fasciitis is a common condition and for most part very treatable. Treatment should begin with conservative management which should include heel cord and plantar fascia stretching excercises. NSAIDs and orthotics can be supplemented as and when necessary. Night splints can be used but are cumbersome and have as low compliance. If symptoms have not improved after these options, injections may be considered. If symptoms still continue to persist for 6 months or more then ESWT or radiation can be attempted. Surgical release is typically a last resort in cases of plantar fasciitis.

\section{References}

1. KBTJF. Economic burden of plantar fasciitis treatment in the United States. American journal of orthopedics (Belle Mead, N.J.), 2010.

2. Lee Wc Fau, Wong WY, Wong Wy Fau, Kung E, Kung E Fau, Leung AKL, Leung AK. Effectiveness of adjustable dorsiflexion night splint in combination with. $\mathbf{J}$ Rehabil Res Dev, 2012.

3. Dyck DD, Boyajian-O'Neill LA. Plantar fasciitis. Clinical Journal of Sport Medicine, 2004.

4. Thomas JL, Christensen JC, Kravitz SR, Mendicino RW, Schuberth JM, Vanore JV et al. The Diagnosis and Treatment of Heel Pain: A Clinical Practice GuidelineRevision 2010. J Foot Ankle Surg, 2010.

5. Young CC, Rutherford DS, Niedfeldt MW. Treatment of plantar fasciitis. American Family Physician, 2001.

6. Malliaropoulos N, Crate G, Meke M, Korakakis V, Nauck T, Lohrer $\mathrm{H}$ et al. Success and Recurrence Rate after Radial Extracorporeal Shock Wave Therapy for Plantar Fasciopathy: A Retrospective Study. Biomed Res Int, 2016;

7. Draghi F, Gitto S, Bortolotto C, Draghi AG, Ori Belometti G. Imaging of plantar fascia disorders: findings on plain radiography, ultrasound and magnetic resonance imaging. Insights into Imaging, 2017.

8. Jha R, Uprety S, Shah L. Functional Outcome in Patients with Chronic Plantar Fasciitis Treated with Plantar Fascia Stetching VS Tendoachilles Stretching Exercises. J Inst Med, 2013.

9. Crawford F, Thomson CE. Interventions for treating plantar heel pain. In: Cochrane Database of Systematic Reviews, 2003.

10. Li Z, Yu A, Qi B, Zhao Y, Wang W, Li P et al. 
Corticosteroid versus placebo injection for plantar fasciitis: A meta-analysis of randomized controlled trials. Exp Ther Med, 2015.

11. Akoahin E, Doğruyol D, Yüksel HY, Hapa O, Doğan Ö, Çelebi L et al. The comparison of the eVect of corticosteroids and platelet-rich plasma (PRP) for the treatment of plantar fasciitis. Arch Orthop Trauma Surg, 2012.

12. Wheeler PC. The role of autologous blood injections in the treatment for patients with chronic plantar fasciitis-A case series and longer-term follow-up. Int. Musculoskelet Med, 2015.

13. Canyilmaz E, Canyilmaz F, Aynaci O, Colak F, Serdar L, Uslu GH et al. Prospective randomized comparison of the effectiveness of radiation therapy and local steroid injection for the treatment of plantar fasciitis. Int. J Radiat Oncol Biol Phys, 2015.

14. Monteagudo M, Maceira E, Garcia-Virto V, Canosa R. Chronic plantar fasciitis: Plantar fasciotomy versus gastrocnemius recession. Int. Orthop, 2013. 